\title{
AUTOMATED BRAIN TUMOR SEGMENTATION IN MR IMAGES USING A HIDDEN MARKOV CLASSIFIER FRAMEWORK TRAINED BY SVD-DERIVED FEATURES
}

\author{
Fazel Mirzaei ${ }^{1}$, Mohammad Reza Parishan ${ }^{2}$, Mohammadjavad Faridafshin ${ }^{3}$, Reza Faghihi ${ }^{4}$ and \\ Sedigheh Sina ${ }^{5}$ \\ ${ }^{1,2,3}$ Department of Medical Radiation Engineering, Shiraz University, Iran \\ ${ }^{4,5}$ Radiation Research Center, Shiraz University, Shiraz, Iran
}

\begin{abstract}
Interpreting brain MR images are becoming automated, to such extent that in some cases "all" the diagnostic procedure is done by computers. Therefore, diagnosing the patients is done by a comparably higher accuracy. Computer models that have been trained by a priori knowledge act as the decision makers. They make decisions about each new image, based on the training data fed to them previously. In case of cancerous images, the model picks that image up, and isolates the malignant tissue in the image as neatly as possible. In this paper we have developed an unsupervised learning system for automatic tumor segmentation and detection that can be applied to low contrast images.
\end{abstract}

Keywords:

Image Segmentation, Hidden Markov Model, Singular Value decomposition, Wavelet Analysis

\section{INTRODUCTION}

Recent developments in Magnetic Resonance Imaging (MRI) have led the imaging society to considerable results in both anatomical and functional visualization and localization of different human organs. MRI is the favorite imaging modality for oncologists to do research on Image-Guided Therapy (IGT), analyze and detect brain tumors [2]. In addition, researches on brain tumor segmentation are carried out mostly by using MR images due to high ability of this modality in visualizing the brain organs, especially at the presence of contrast agents [1].

Immethodical cell growth is the main reason for brain tumors to emerge [2]. IGT-based brain tumor detection and elimination relies highly on efficient tumor localization in images. Tumors are detected either manually or automatically. In automatic brain tumor detection systems, image processing and machine learning algorithms are employed [4][5].

Manual tumor detection is a time consuming process and could even be risky if the tumor size is too small [5]. This issue is addressed by developing computer programs to automatically analyze the images for detecting possible malignancies, and several machine learning and image processing algorithms for decision making, information extraction and brain tumor segmentation have been proposed [4][6].

In brain tumor segmentation, two clusters are isolated in the image by labeling the pixels as either healthy or cancerous by calculating some features [7]. The conventional features for classification are the pixel intensity, depth, color and texture [8]. Similarity measures like distance between two feature vectors or their normalized inner product can also be used as pixel cluster identifiers. Pixel-based image segmentation techniques are conducted in supervised and unsupervised ways [8][5].
In this article, we have studied the application of Hidden Markov Random Field (HMRF) unsupervised pixel-based modelling and Singular Value Decomposition (SVD) feature extraction method together with wavelet image analysis in segmenting the brain tumor MR images.

The structure of the paper in as follows: in section 2 the implemented theories and the flowchart of the work are presented, in section 3 the results are quantified evaluated and a discussion about the results is given in section 4 .

\section{MATERIALS AND METHODS}

\subsection{SINGULAR VALUE DECOMPOSITION (SVD)}

Extracting image features is a crucial step in pattern recognition problems. Despite the conventional visual features mentioned in the last section, more complex features like algebraic, statistical and transform coefficient features can also be defined for images. In [11], "algebraic" features are studied and proved as insensitive to image noise and invariant to geometric change, which makes them useful for object recognition in images. Singular values are algebraic features defined for images and are extracted by SVD algorithm.

Using the following Eqn, the image $I-m \times n$ rectangular matrix with rank $k$ is transformed into a diagonal matrix by introducing three diagonal matrices $U_{m \times k}, V_{k \times n}$, and $\sum_{k \times k}$, where, $\sum_{k \times k}$ contains positive real entries and is a diagonal matrix in which the diagonal elements are the singular values of the image $I$.

$$
I=U \Sigma V^{T}
$$

In this study, the first element of $U$ and the first two diagonal elements of $\sum$ are chosen as features. These features are then normalized throughout the entire database of brain MR images.

\subsection{HIDDEN MARKOV RANDOM FILED (HMRF)}

Hidden Markov Random Field (HMRF) is an unsupervised probabilistic way to model images. Modeling in this way is based on a data generation process called "Markov Chain", and the obtained model is a "Hidden Markov Model (HMM)" [12] [13]. In an HMRF model, images are represented by matrices with probabilistic dependencies between pixels - i.e. nodes. These dependencies can be depicted using an undirected graphical model, in which the labels are not known previously and are predicted based on the status of the neighborhood pixels by a probability distribution, and thus are "hidden" from us[14] [15]. Therefore, each pixel is represented as a node $P_{i}$ associated with a label $X_{i}$ generated from a probability distribution $P(x)$ (Fig.1) [12]. 


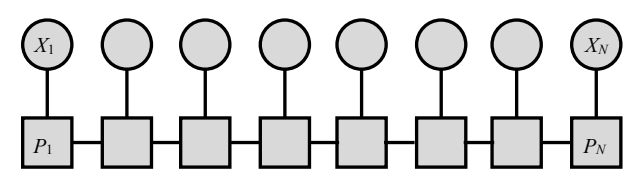

Fig.1. Undirected graphical representation of pixels $P_{1}$ to $P_{N}$ with corresponding labels $X_{1}$ to $X_{N}$

If $X$ denotes the set of unobserved variables i.e. the labels and $Y$ denotes the set of observed variables i.e. pixel intensities, the posterior distribution $P(X=x \mid Y=y)$ is computed according to Bayes formula (Eq.(2)). In Eq.(2), $P(X=x \mid \omega)$ is the prior distribution of the set of labels conditioned on the vector of parameters $\omega$, and $P(Y=y \mid X=x, \omega)$ is the likelihood probability, and the denominator is a normalizing constant which makes the posterior probability of all the members of set $X$ to sum to 1 [16]. After knowing the posterior probability for each pixel (or pixel clique), decision is taken about the unobserved pixel using Maximum a Posteriori (MAP) criteria [17].

$$
P(X=x \mid Y=y, \omega)=(P(Y=y \mid X=x, \omega) P(X=x \mid \omega)) /(P(Y))
$$

By using the MAP criteria, the unobserved label $\hat{X}$ of a pixel is inferred by the maximizing the posterior probability. In order to fully define the argument in Eq.(3), the prior and the likelihood probability distributions must be characterized in advance. Since the prior distribution is a Markov Random Field (MRF) it can be equivalently characterized by a Gibbs distribution, according to Hamersley-Clifford theorem [18]. Moreover, the likelihood is assumed to follow a Gaussian distribution with $\omega$ as the vector of mean and variance. The effectiveness of Gibbs distributions as priors for image processing tasks has been studied and proved useful in a number of studies [18] - [21].

$$
\hat{x}=\operatorname{argmax}(P(Y=y \mid X=x, \omega) P(X=x \mid \omega))
$$

Gibbs distribution [20] is characterized by an energy function $\mathrm{U}$ and a partition function $Z$ which is a normalizing constant; and the likelihood is represented by a Gaussian with parameter vector $\omega=\left\{\sigma_{1}, \mu_{1}\right\}$. The optimal value of the parameters specifying the likelihood distribution is calculated using ExpectationMaximization (EM) algorithm.

$$
\begin{gathered}
P(X=x)=\frac{1}{N} \exp (-U(x, \omega)) \\
P(Y=y \mid X=x, \omega)=\frac{1}{\sqrt{2 \pi \sigma_{x}^{2}}} \exp \left(-\frac{\left(y-\mu_{x}\right)^{2}}{2 \sigma_{x}^{2}}\right)
\end{gathered}
$$

The Hidden Markov Random Field (HMRF) together with EM algorithm briefly discussed in this section is implemented for image segmentation according to [22].

\subsection{WAVELET TUMOR SEGMENTATION}

In the last section, HMRF-EM algorithm was utilized to detect and locate the cancerous pixels in image [18] [22]. This section addresses the tumor isolation task, in which the image is segmented into cancerous and non-cancerous regions [22]. To enhance the corners and edges more efficiently, high-pass and edge enhancement filters are applied to the image respectively. By performing the wavelet transform in the next step, the image is decomposed into 8 levels of wavelet [8] [13]. The tumor is isolated from the rest of the brain area in the image by allowing the values in the lower band to reset the highest levels of wavelet decomposition to zero. The image is then reconstructed by taking the inverse wavelet transform of the multilevel wavelet structure.

The wavelet transform is generalized to two dimensions by carrying out one-dimensional transform on each image dimension. In each step, four sub-bands approximating sub-band $\left(A_{j}\right)$, horizontal detail sub-band $\left(D_{j}^{h}\right)$, vertical detail sub-band $\left(D_{j}{ }^{v}\right)$ and diagonal detail sub-band $\left(D_{j}^{d}\right)$ are produced by approximating the sub-band of the last step $\left(A_{j}-1\right)$. Then, the approximated subband represents the image background with all of it's lowfrequency fluctuations, while horizontal/vertical/diagonal detail sub-bands represent the high pass fluctuations in horizontal /vertical/diagonal axes of the image[13] [17] (Fig.2).

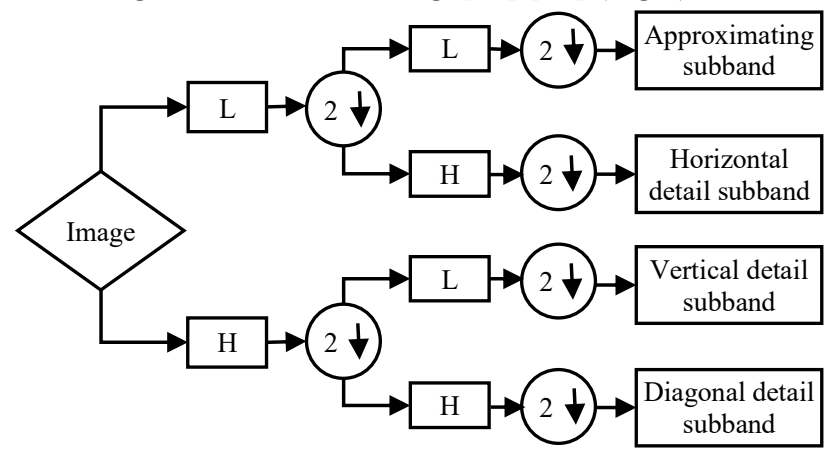

Fig.2. 2D Wavelet Decomposition

In the next step, Otsu's image threshold is computed to finally segment the image already enhanced by wavelet transform. By thresholding, some irregularities are segmented in addition to the tumor; therefore, a threshold for the number of connected pixels is specified and the connected bunch of pixels consisting less pixels than the threshold are eliminated from the image [23].

\subsection{PROPOSED METHOD}

This study is followed through the flowchart of Fig.3. Briefly, the database and test input images are processed for noise reduction, resizing, and blocking in the first block to be ready for feature extraction. The features from database images are fed into an HMRF classifier for training. The classifier is then tested by the test input image features (namely "input images" in Fig.3). The input images are divided into cancerous and non-cancerous groups. The cancerous images are further segmented by computing wavelet coefficients and at last, the tumor completely stands out in the output binary image after being de-noised by Otsu's method.

\section{RESULTS AND EVALUATION}

In this study, the steps illustrated in Fig. 3 are performed on a standard database of brain MR images. The method can be divided into two parts; the localization and the isolation phase of the tumor. First, the images are pre-processed and algebraic feature vectors are extracted using SVD algorithm. Then, HMRF classifier is trained using the extracted feature vectors and applied on the images in order to decide on the existence of the tumor and locate it if so. In the second phase, the proposed segmentation algorithm is applied on the images diagnosed as cancerous to further analyze the cancerous images by the wavelet transform 
and isolate the cancerous area from the rest of the image. In Fig.4, the results of applying the method on five test images are depicted. In addition to the proposed method using HMRF modeling, conventional MRF, morphology and manual segmentation results are illustrated in Fig.5. As can be seen from the results obtained by MRF, HMRF, and Morphological Tech segmentation methods and comparing them to the ground truth, HMRF in both three test images has shown better performance in detecting the tumor.
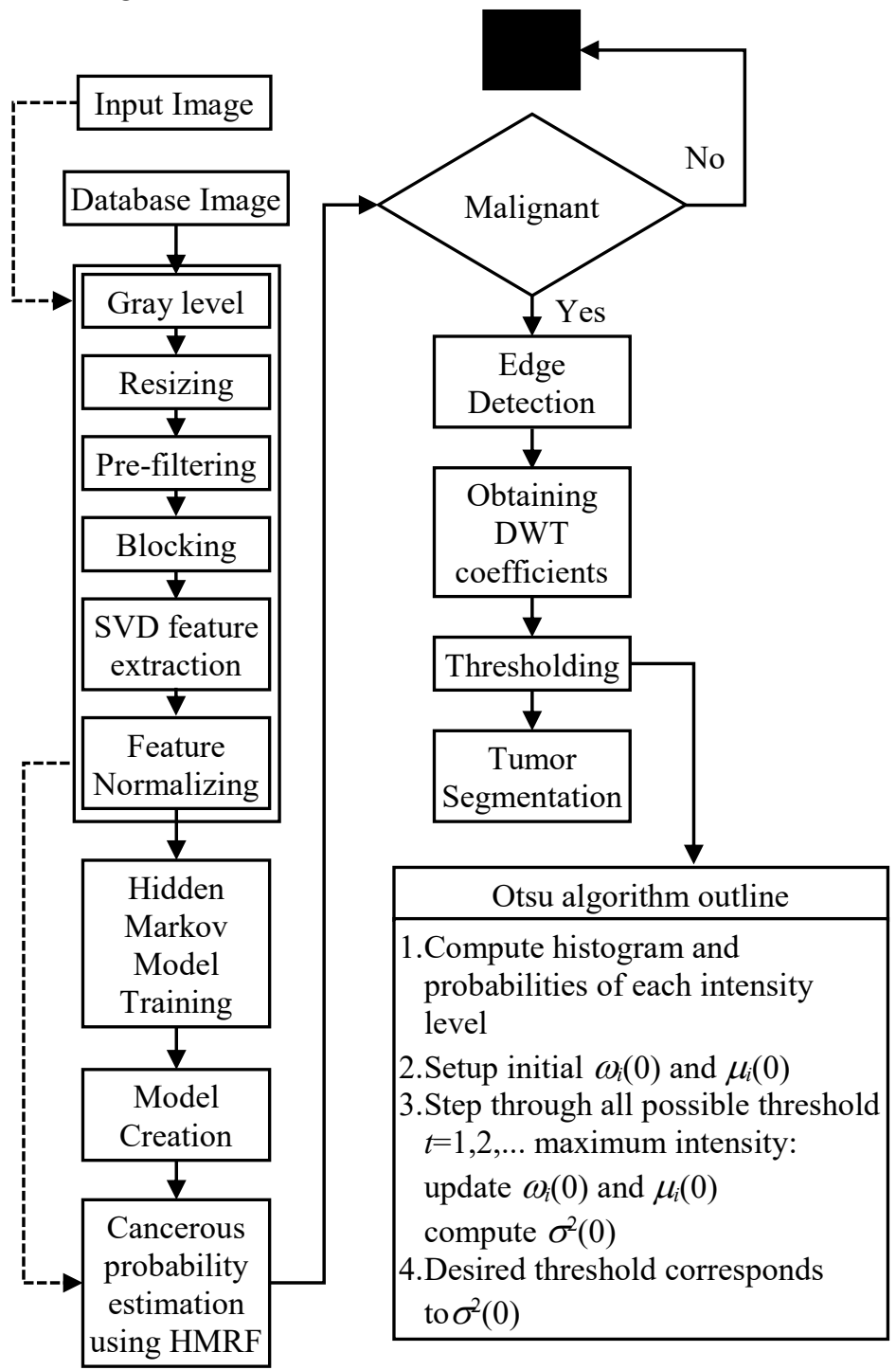

Fig.3. The Proposed Algorithm

To evaluate the results quantitatively, a number of metrics are used in this study; therefore, Volume Overlap Ratio (VOR), Recognition Rate $(R R)$, True Negative Rate (TNR), True Positive Rate (TPR), Accuracy, Sensitivity and Specificity are calculated for result evaluation (Eq.(6) to Eq.(10)).

$$
V O R=\frac{I_{c} \cup I_{r}}{I_{c} \cap I_{r}}
$$

where, $I_{c}$ and $I_{r}$ are the images segmented by the algorithm and radiologist respectively.

$$
\begin{aligned}
\text { Accuracy }= & (T P+T N) /(T P+F P+F N+T N) \\
& T P R=T P /(T P+F P)
\end{aligned}
$$

$$
\begin{aligned}
& \text { Sensitivity }=T P /(T P+F N) \\
& \text { Specificity }=T N /(T N+F P)
\end{aligned}
$$

\begin{tabular}{|c|c|c|c|c|c|}
\hline & $\begin{array}{c}\text { Test } \\
\text { image } 1\end{array}$ & $\begin{array}{c}\text { Test } \\
\text { image } 2\end{array}$ & $\begin{array}{c}\text { Test } \\
\text { image } 3\end{array}$ & $\begin{array}{c}\text { Test } \\
\text { image } 4\end{array}$ & $\begin{array}{c}\text { Test } \\
\text { image } 5\end{array}$ \\
\hline $\begin{array}{l}\text { Original } \\
\text { Images }\end{array}$ & & & & & \\
\hline $\begin{array}{c}\text { Detected } \\
\text { Edges }\end{array}$ & & & & & \\
\hline Segmente & & 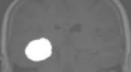 & & & \\
\hline $\begin{array}{c}\text { Extracted } \\
\text { Tumors }\end{array}$ & & & & & \\
\hline
\end{tabular}

The measures are calculated for the whole database. The aggregated results are in Table.1, and the specific results for the test images in Fig.5 are in Table.2.

Fig.4. Qualitative results of applying the segmentation method on five of brain MR images database

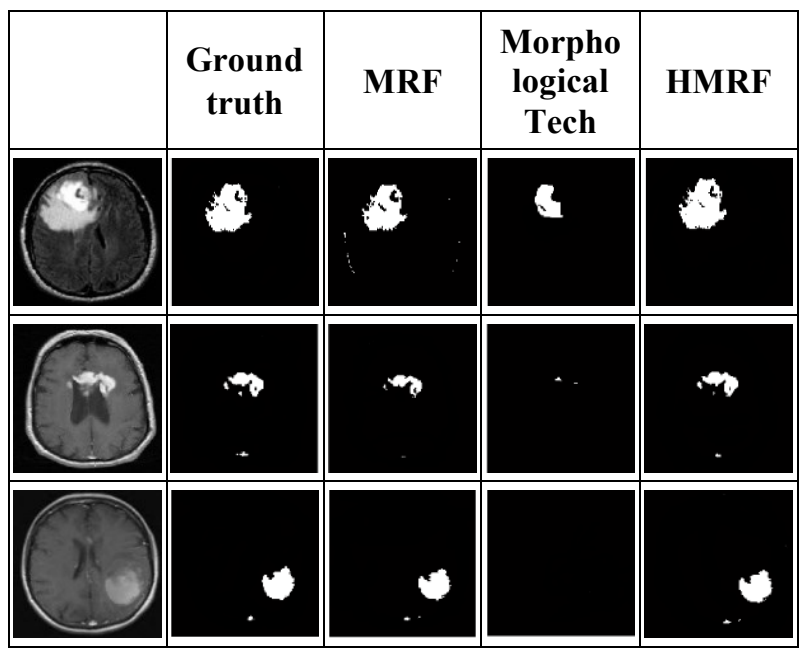

Fig.5. MRF, Morphological Tech and HMRF qualitative comparison

Table.1. Quantitative results for all images in the database

\begin{tabular}{|l|c|c|c|}
\hline & Min & Max & Mean \\
\hline VOR (\%) & 63.1 & 96.87 & 78.35 \\
\hline ACC (\%) & 80.2 & 97.32 & 92.53 \\
\hline TPR (\%) & 89.58 & 98.17 & 95.06 \\
\hline
\end{tabular}

Table.2. Quantitative results for each of the five images

\begin{tabular}{|l|l|l|l|l|} 
Image1 & Image 2 & Image 3 & Image4 & Image 5 \\
\hline
\end{tabular} 


\begin{tabular}{|c|c|c|c|c|c|}
\hline VOR (\%) & 94.52 & 91.17 & 96.28 & 90.66 & 94.35 \\
\hline ACC (\%) & 90.15 & 96.73 & 92.11 & 94.42 & 94.64 \\
\hline TPR (\%) & 97.17 & 97.08 & 94.51 & 95.89 & 93.16 \\
\hline Sensitivity (\%) & 92.61 & 94.23 & 90.14 & 95.43 & 91.19 \\
\hline Specificity (\%) & 93.34 & 95.1 & 92.13 & 92.62 & 94.58 \\
\hline
\end{tabular}

\section{DISCUSSIONS}

Statistical models have found great application in image processing since long time ago. Among different branches of image processing, statistical models have found great use in medical image segmentation [3], [18], [21]. In this study, a method for location and isolation the tumor in brain MR images is proposed. This method consists of two phases; location and isolation. At the first step, a model based on HMRF utilizing SVD feature extraction algorithm is developed to separate cancerous and non-cancerous images and locate the tumor in cancerous ones. For further isolation the tumor from the background, wavelet analysis is performed through direct and inverse wavelet transforms. The HMRF-Wavelet method is performed on brain MR images, and the performance of the algorithm is evaluated both qualitatively and quantitatively. In the results section, the performance of proposed algorithm is presented and, as the results show, the proposed algorithm is successful in isolating the tumor. In addition, some criteria are used to examine the results quantitatively. The mean amount of the VOR, ACC and TPR is, $78.35,92.53$ and 95.06 respectively. The minimum and maximum values for each of the evaluation parameters are determined by varying the classifier parameters and training observations.

Of course, the proposed algorithm also has some disadvantages that can be pointed out as follows:

\section{Advantages:}

- Low sensitivity to inhomogeneity.

- Capable of being applied to difficult situations.

- Unsupervised learning.

\section{Disadvantages:}

- Computational Complexity.

- Slow learning phase.

- There are lots of parameters that must be controlled.

\section{CONCLUSION}

In this study, a method is proposed for segmenting the brain MR images. A Hidden Markov Random Field (HMRF) model is trained using features extracted by Singular Value Decomposition (SVD) algorithm, and the location of the tumor is extracted accordingly. Then, wavelet analysis is performed and the image is decomposed into eight levels of wavelet for better isolation of the tumor from the background. The performance of proposed method is evaluated and results are shown. The method implemented in this paper recognizes the cancerous pixels more sufficiently than MRF and Morphological Tech methods.

\section{REFERENCES}

[1] N. Gordillo, E. Montseny and P. Sobrevilla, "State of the Art Survey on MRI Brain Tumor Segmentation", Magnetic Resonance Imaging, Vol. 31, No. 8, pp. 1426-1438, 2013.

[2] B. Likar, P. Markelj and D. Tomaz, "A review of 3D / 2D Registration methods for Image-Guided Interventions", Medical Image Analysis, Vol. 16, No. 3, pp. 642-661, 2012.

[3] E.H. Guerrout, R. Mahiou and S. Ait-Aoudia, "Medical Image Segmentation using Hidden Markov Random Field A Distributed Approach", Proceedings of $3^{\text {rd }}$ International Conference on Digital Information Processing and Communications, pp. 423-430, 2013.

[4] S. Faisan, L. Thoraval, J. Armspach and F. Heitz, "Unsupervised Learning and Mapping of Active Brain Functional MRI Signals Based on Hidden Semi-Markov Event Sequence Models", IEEE Transactions on Medical Imaging, Vol. 24, No. 2, pp. 263-276, 2005.

[5] C.A.S. Sergio Pereira, Adrino Pinto and Victor Aves, "Brain Tumor Segmentation using Convolutional Neural Networks in MRI Images", IEEE Transactions on Medical Imaging, Vol. 35, No. 5, pp. 1240-1251, 2016.

[6] M.K.N. Nabizadeh and M. Dorodchi, "Automatic Tumor Lesion Detection and Segmentation using Modified Winnow Algorithm", Proceedings of IEEE $12^{\text {th }}$ International Symposium on Biomedical Imaging, pp. 212218, 2015.

[7] I.G. Ivan Cabria, "Automated Localization of Brain Tumors in MRI using Potential-K-Means Clustering Algorithm", Proceedings of $12^{\text {th }}$ Conference on Computer and Robot Vision, pp. 1-4, 2015.

[8] H. Choi, R. G. Baraniuk and S. Member, "Multiscale Image Segmentation using Wavelet-Domain Hidden Markov Models", IEEE Transactions on Image Processing, Vol. 10, No. 9, pp. 1309-1321, 2001.

[9] S.L. Jui et al., "Brain MR Image Tumor Segmentation with 3-Dimensional Intracranial Structure Deformation Features", IEEE Intelligent Systems, Vol. 31, No. 2, pp. 1-9, 2015.

[10] I. Ali and C. Direko, "Review of MRI-based Brain Tumor Image Segmentation using Deep Learning Methods", Proceedings of $12^{\text {th }}$ International Conference on Application of Fuzzy Systems and Soft Computing, pp. 317324, 2016.

[11] Z. Hong, "Algebraic Feature Extraction of Image for Recognition”, Pattern Recognition, Vol. 24, No. 3, pp. 211219, 1991.

[12] Andrew Blake, Pushmeet Kohli and Carsten Rother, "Markov Random Fields for Vision and Image Processing", MIT Press, 2011.

[13] H. Miar-Naimi and P. Davari, "A New Fast and Efficient HMM-Based Face Recognition System using a 7-State HMM Along with SVD Coefficients", Iranian Journal of Electrical and Electronic Engineering, Vol. 4, No. 1, pp. 4657, 2008.

[14] T.L. Griffiths and A. Yuille, "Technical Introduction: A Primer on Probabilistic Inference", Master Thesis, Department of Statistics, University of California, pp. 1-57, 2008.

[15] T. Chen and T.S. Huang, "Region Based Hidden Markov Random Field Model for Brain MR Image Segmentation", 
International Journal of Computer and Information Engineering, Vol. 1, No. 4, pp. 1129-1132, 2007.

[16] J.L. Marroquin, E.A. Santana and S. Botello, "Hidden Markov Measure Field Models for Image Segmentation", IEEE Transactions on Pattern Analysis and Machine Intelligence, Vol. 25, No. 11, pp. 1380-1387, 2003.

[17] R. Azmi and N. Norozi, "A New Markov Random Field Segmentation Method for Breast Lesion Segmentation in MR Images", Journal of Medical Signals and Sensors, Vol. 1, No. 3, pp. 156-164, 2011.

[18] Y. Zhang, M. Brady and S. Smith, "Segmentation of Brain MR Images Through a Hidden Markov Random Field Model and the Expectation-Maximization Algorithm", IEEE Transactions on Medical Imaging, Vol. 20, No. 1, pp. 45-57, 2001.

[19] E. Levitan, M. Chan and G.T. Herman, "Image-Modeling Gibbs Priors", Graphical Models and Image Processing, Vol. 57, No. 2, pp. 117-130, 1995.
[20] H. Derin and H. Elliot, "Modeling and Segmentation of Noisy and Textured Images using Gibbs Random Fields", IEEE Transactions on Pattern Analysis and Machine Intelligence, Vol. 9, No. 1, pp. 39-55, 1987.

[21] T. Zhang, Y. Xia and D. Dagan, "Hidden Markov Random Field Model based Brain MR Image Segmentation using Clonal Selection Algorithm and Markov Chain Monte Carlo Method", Biomedical Signal Processing and Control, Vol. 12, pp. 10-18, 2014.

[22] R.S.A. Padma Nanthagopal, "Wavelet Statistical Texture Features-based Segmentation and Classification of Brain Computed Tomography Images", IET Image Processing, Vol. 7, No. 1, pp. 25-32, 2013.

[23] H.S. Abdulbaqi and A.F. Omar, "Detecting Brain Tumor in Magnetic Resonance Images using Hidden Markov Random Fields and Threshold Techniques", Proceedings of IEEE Student Conference on Research and Development, pp. 1617,2014 